\title{
RADICALS OF SEMIGROUP RINGS $\dagger$
}

\author{
by JULIAN WEISSGLASS $\ddagger$
}

(Received 20 March, 1968; revised 9 December, 1968)

1. Introduction. Let $\overline{R D}$ denote the contracted semigroup ring of the completely 0 -simple semigroup $D$ over the ring $R$. The Rees structure theory of completely 0 -simple semigroups is used to obtain necessary and sufficient conditions that $\overline{R D}$ have zero radical (Theorem 3.8). By using Amitsur's construction of the upper $\pi$-radical [1], we are able to treat the Jacobson, Baer (prime), Levitzki (locally nilpotent) and possibly the nil radicals simultaneously. Our results generalize a theorem of Munn [6] on semigroup algebras of finite 0 -simple semigroups.

\section{Preliminaries.}

(A) RADICALS. Let $\pi$ be a property of rings. If the ring $R$ has property $\pi$ we say $R$ is a $\pi$-ring. We call an ideal a $\pi$-ideal if as a ring it is a $\pi$-ring. We say the ring $R$ is $\pi$ semisimple if it has no nonzero $\pi$-ideals.

Following Amitsur [1], we define a sequence of ideals of $R, U_{\lambda}=U_{\lambda}(R)$, for ordinals $\lambda$ as follows:

(1) $U_{0}=\{0\}$.

(2) If $\lambda$ has predecessor $\lambda-1, U_{\lambda}$ is the sum of all ideals $P$ of $R$ containing $U_{\lambda-1}$ such that $P / U_{\lambda-1}$ is a $\pi$-ring.

(3) If $\lambda$ is a limit ordinal, $U_{\lambda}$ is the sum of all $U_{v}$ for $v<\lambda$.

The upper $\pi$-radical $\mathscr{U}(R, \pi)=\mathscr{U}(R)$ is defined to be the limit ideal of this sequence; i.e. $\mathscr{U}(R)$ is the minimal ideal $U_{\lambda}$ such that $U_{\lambda}=U_{\lambda+1}$. It is clear that $\mathscr{U}(R)=\{0\}$ if and only if $R$ is $\pi$-semisimple.

The property $\pi$ is called an H-property if homomorphic images of $\pi$-rings are $\pi$-rings. We include the zero homomorphism. Thus, if $\pi$ is an H-property, the ring consisting of $\{0\}$ is a $\pi$-ring.

The following definition will be useful.

Definition 2.1. A property $\pi$ of rings is left inductive if

(i) $\pi$ is an H-property,

(ii) $\pi$-semisimple rings have no left $\pi$-ideals,

(iii) left ideals of $\pi$-rings are $\pi$-rings.

Remark 2.2. Amitsur [1, Theorem 3.1] shows that for H-properties condition (ii) is equivalent to: the upper $\pi$-radical of a ring contains every left $\pi$-ideal of the ring.

† This paper is part of the author's doctoral dissertation at the University of Wisconsin written under the direction of Professor Hans Schneider.

$\ddagger$ The author was supported in part by research grant NSF-GP-3993.

F 
Some well known radicals are obtained in the following way:

1. Left quasi-regular. By definition, $R$ is a $\pi$-ring if for every $a \in R$ there exists $x$ such that $x \circ a=a+x-x a=0$. The upper $\pi$-radical $\mathscr{U}(R)$ is then the Jacobson radical of $R$, which we shall denote by $\mathscr{J}(R)$. It is well known that left quasi-regularity is left inductive.

2. Nil. By definition, $R$ is a $\pi$-ring if, for every $a \in R$, there exists an integer $n=n(a)$ such that $a^{n}=0$. The upper $\pi$-radical is then the (upper) nil radical. We shall denote it by $\mathscr{N}(R)$. Although nil satisfies 2.1(i) and 2.1(iii) it is not known whether a ring with no nil ideals can have a nil left ideal.

3. Locally nilpotent. By definition, $R$ is a $\pi$-ring if for every finite subset $A$ of $R$ there exists an integer $n=n(A)$ such that $A^{n}=\{0\}$. We shall denote the upper $\pi$-radical in this case by $\mathscr{L}(R)$. It is called the locally nilpotent, or Levitzki, radical of $R$. Amitsur has shown that locally nilpotent (semi-nilpotent in his terminology) is a left inductive property. ([2], Theorems 1.3 and 2.1.)

4. Nilpotent. By definition, $R$ is a $\pi$-ring if there exists an integer $n$ such that $R^{n}=\{0\}$. The upper $\pi$-radical will be denoted by $\mathscr{P}(R) . \mathscr{P}(R)$ is commonly called Baer's radical or the prime radical (since it is the intersection of all the prime ideals of $R$ ). Nilpotent is a left inductive property.

Note that all four of the above properties $\pi$ satisfy:

$$
R^{2}=\{0\} \text { implies that } R \text { is a } \pi \text {-ring. }
$$

We call $\pi$ a Z-property if it satisfies Condition (Z). We also observe that all four properties satisfy the following condition.

Condition 2.3. If $A$ is an ideal in a $\operatorname{ring} B$ such that $A$ and $B / A$ are $\pi$-rings, then $B$ is a $\pi$-ring.

We now establish some preliminary results on the relationship between the radical of a left ideal in a ring and the radical of the ring.

Lemma 2.4. Let $\pi$ be left inductive and let $R$ be a left ideal of a ring $S$.

(i) If $L$ is a left $\pi$-ideal of $R$, then $R L \subseteq \mathscr{U}(S)$.

(ii) If $Q$ is a left $\pi$-ideal of $S$, then $Q \cap R \subseteq \mathscr{U}(R)$.

Proof. (i) $R L$ is a left ideal of $S$. Also $R L \subseteq L$, so that $R L$ is a left ideal of $L$. By Definition 2.1(iii), $R L$ is a $\pi$-ring. Hence, by Remark 2.2, $R L \subseteq \mathscr{U}(S)$.

(ii) $Q \cap R$ is a left ideal of $Q$. Hence $Q \cap R$ is a $\pi$-ring. But $Q \cap R$ is also a left ideal of $R$. Hence, by Remark 2.2, $Q \cap R \subseteq \mathscr{U}(R)$.

Remarks 2.5. (i) Stronger assumptions on $\pi$ permit the specific determination of the radical of a left ideal. See Amitsur [1].

(ii) In Lemma 2.4(i) it may not be true that $L$ is contained in $\mathscr{U}(S)$ even if $L$ is a (twosided) ideal of $R$. For example, let $S$ be the ring of $2 \times 2$ matrices over a field. Let $R$ be the left ideal of $S$ consisting of matrices with second column zero. Let $L$ be the ideal of $R$ con- 
sisting of matrices with upper left hand corner zero. Let $\mathscr{U}=\mathscr{J}$, the Jacobson radical. Then $\mathscr{J}(S)=0$, but $L$ is a quasi-regular ideal of $R$.

If $M$ is a left (right) $T$-module, then we denote the left (right) annihilator of $M$ in $T$ by $\mathfrak{N}\left({ }_{T} M\right)\left(\mathfrak{N}\left(M_{T}\right)\right)$. In particular, $\mathfrak{N}\left(R_{R}\right)$ is the right annihilator of $R$ in $R$.

THEOREM 2.6. Let $\pi$ be left inductive and let $R$ be a left ideal of a ring $S$.

(i) If $\mathfrak{Y}\left(R_{R}\right)=\{0\}$, then $\mathscr{U}(S)=\{0\}$ implies that $\mathscr{U}(R)=\{0\}$.

(ii) If $\mathfrak{Y}\left({ }_{S} R\right)=\{0\}$, then $\mathscr{U}(R)=\{0\}$ implies that $\mathscr{U}(S)=\{0\}$.

Hence, if $\mathfrak{N}\left(R_{R}\right)=\{0\}$ and $\mathfrak{N}\left({ }_{S} R\right)=\{0\}$, then $\mathscr{U}(R)=\{0\}$ if and only if $\mathscr{U}(S)=\{0\}$.

Proof. (i) Assume that $\mathscr{U}(S)=\{0\}$. By Lemma 2.4(i), any $\pi$-ideal of $R$ annihilates $R$ on the right. But $\mathfrak{Y}\left(R_{R}\right)=\{0\}$; hence there are no nonzero $\pi$-ideals in $R$, so that $\mathscr{U}(R)=\{0\}$.

(ii) Assume that $\mathscr{U}(R)=\{0\}$. By Lemma 2.4(ii), if $Q$ is a $\pi$-ideal of $S, Q \cap R=\{0\}$. But $Q R \subseteq Q \cap R$ if $Q$ is an ideal of $S$. Hence any $\pi$-ideal of $S$ annihilates $R$ on the left. But $\mathfrak{Q}\left({ }_{S} R\right)=\{0\}$, so that $S$ has no $\pi$-ideals and $\mathscr{U}(S)=\{0\}$.

(B) COMPLETELY 0-SIMPLE SEMIGROUPS. A subset $A$ of a semigroup $D$ is a left (right) ideal if $D A \subseteq A(A D \subseteq A) . A$ is an ideal if it is both a left and a right ideal. A semigroup $D$ with a zero element 0 is called 0 -simple if its only ideals are $\{0\}$ and $D$ and $D^{2} \neq\{0\}$. The set of idempotents of a semigroup may be partially ordered by defining $e \leqq f$ to mean $e f=f e=e$. (Cf. [4], Section 1.8.) An idempotent $f$ is said to be primitive if $f \neq 0$ and $e \leqq f$ implies that $e=0$ or $e=f$. A completely 0 -simple semigroup is a 0 -simple semigroup with a primitive idempotent. Clearly, a finite 0 -simple semigroup is completely 0 -simple.

Let $T$ be a semigroup and let $I, \Lambda$ be sets. Define $M(T ; I, \Lambda)$ to be the set of all mappings $A: I \times \Lambda \rightarrow T$. Denote the image of $(i, \lambda)$ under $A$ by $a_{i \lambda}$ and write $A=\left(a_{i \lambda}\right)$. The elements of $M(T ; I, \Lambda)$ are called $I \times \Lambda$ matrices over $T$. The element $a_{i \lambda}$ is called the $(i, \lambda)$-entry of $A$. Let $T$ be a semigroup with zero. Let $A$ be an $I \times \Lambda$ matrix over $T$ and $B$ a $\Lambda \times I$ matrix over $T$. Define $A B$ to be the $I \times I$ matrix $C=\left(c_{i j}\right)$, where $c_{i j}=\sum_{\lambda} a_{i \lambda} b_{\lambda j}$ if, for every $(i, j)$, at most one summand in $\sum_{\lambda} a_{i \lambda} b_{\lambda j}$ is nonzero. Otherwise the product is not defined.

The group with zero $G^{0}$ is just the semigroup obtained by adjoining a zero to the group $G$. A Rees $I \times \Lambda$ matrix over the group with zero $G^{0}$ is an $I \times \Lambda$ matrix over $G^{0}$ having at most one nonzero entry. Let $P=\left(p_{\lambda i}\right)$ be an arbitrary but fixed $\Lambda \times I$ matrix over $G^{0}$. Then $A P$ and $P A$ are defined for every Rees $I \times \Lambda$ matrix $A$ over $G^{0}$. Define the binary operation "." on the set of Rees $I \times \Lambda$ matrices over $G^{0}$ by $A \cdot B=A P B$. If $A$ has $a$ as its nonzero entry in the $(i, \lambda)$-position and $B$ has $b$ as its nonzero entry in the $(j, \mu)$-position, then $A \cdot B$ is the $I \times \Lambda$ matrix having $a p_{\lambda j} b$ as its $(i, \mu)$-entry and zero elsewhere. Hence $A \cdot B$ is a Rees $I \times \Lambda$ matrix over $G^{0}$. It is easily seen that this multiplication is associative. Therefore the set of all Rees $I \times \Lambda$ matrices over $G^{0}$ is a semigroup with respect to the multiplication ".". This semigroup is denoted by $\mathscr{M}\left(G^{0} ; I, \Lambda ; P\right)$. It is called the Rees $I \times \Lambda$ matrix semigroup over the group with zero $G^{0}$ having sandwich matrix $P$. The construction is important in the characterization of completely 0 -simple semigroups. Rees [8] has shown that a semigroup is completely 0 -simple if and only if it is isomorphic to a Rees matrix semigroup over a group with 
zero with the sandwich matrix $P$ having at least one nonzero entry in each row and column (see also [4]). This last fact enables us to exhibit an isomorphism between the contracted semigroup ring of a completely 0 -simple semigroup and a certain matrix ring.

(C) RINGS OF INFINITE MATRICES. If $T$ is a ring, and so $a$ fortiori a semigroup with zero, $M(T ; I, \Lambda)$, the $I \times \Lambda$ matrices over $T$, can be made into an abelian group. If $a=\left(a_{i \lambda}\right)$ and $B=\left(b_{i \lambda}\right)$, define $A+B$ to be $\left(c_{i \lambda}\right)$, where $c_{i \lambda}=a_{i \lambda}+b_{i \lambda}$. Now let $A$ be an $I \times \Lambda$ matrix over $T$ and let $B$ be a $\Lambda \times I$ matrix over $T$. Define the product $A B$ to be the $I \times I$ matrix $D=\left(d_{i j}\right)$ if $d_{i j}=\sum_{\lambda} a_{i \lambda} b_{\lambda j}$ is defined for every $(i, j)$ (i.e. all but finitely many of the summands are zero). Otherwise the product is undefined. Let $A$ and $C$ be $I \times \Lambda$ matrices over a ring $T$ and let $B$ be a $\Lambda \times I$ matrix over $T$. It may happen that $A B$ and $B C$ are defined but $(A B) C$ and $A(B C)$ are not defined. However, if $(A B) C$ and $A(B C)$ are defined, it is easily seen that $(A B) C=A(B C)$.

An $I \times \Lambda$ matrix $A=\left(a_{i \lambda}\right)$ is column finite if, for every $\lambda \in \Lambda$, there exists a finite subset $N(\lambda) \subseteq I$ such that $a_{i \lambda}=0$ if $i \notin N(\lambda)$. The matrix $A$ is column bounded if the finite subsets $N(\lambda)$ can be chosen to be independent of $\lambda$ (i.e. if there exists a finite subset $N \subseteq I$ such that $a_{i \lambda}=0$ if $\left.i \notin N\right)$. Row finite and row bounded are defined analogously. We say the matrix $A$ is bounded if $a_{i \lambda}=0$ for all but finitely many $(i, \lambda)$. The matrix $A$ is bounded if and only if $A$ is both row and column bounded.

If $A$ is a bounded $I \times \Lambda$ matrix and $P$ is any $\Lambda \times I$ matrix, the products $A P$ and $P A$ are defined. In fact, $A P$ is a column bounded $I \times I$ matrix. Also, if $C$ is a column bounded $I \times I$ matrix and $B$ is a bounded $I \times \Lambda$ matrix, then $C B$ is a bounded $I \times \Lambda$ matrix. Hence, if $A$ and $B$ are bounded $I \times \Lambda$ matrices and $P$ is any $\Lambda \times I$ matrix, the product $(A P) B$ is a bounded $I \times \Lambda$ matrix. Similarly $A(P B)$ is a bounded $I \times \Lambda$ matrix. Since both products are defined, $(A P) B=A(P B)$. Fix a $\Lambda \times I$ matrix $P$ and define a binary operation on the set of all bounded $I \times \Lambda$ matrices by $A \cdot B=A P B$. This operation is seen to be associative and the distributive laws are easily verified. Hence the set of all bounded $I \times \Lambda$ matrices is a ring with respect to $A \cdot B=A P B$. This ring is denoted by $\mathscr{H}_{\rho \gamma}^{*}(T ; I, \Lambda ; P)$. If $\Lambda=I$ and if $P$ is the $I \times I$ identity matrix, we write $M_{\rho \gamma}^{*}(T ; I, I)$ in place of $\mathscr{M}_{\rho \gamma}^{*}(T ; I, I ; P)$. (The notation used is based on that of Patterson [7]. The subscripts $\rho$ and $\gamma$ refer to row and column finiteness. The ${ }^{*}$ indicates that the rows or columns or both, depending on the subscripts appearing, are bounded. We use $\mathscr{M}$ or $M$ depending on whether sandwich multiplication or ordinary multiplication occurs. In particular $M_{\gamma}^{*}(T ; I, I)$ denotes the ring of all column bounded matrices over $T$ under ordinary multiplication. Multiplication of square column bounded matrices is always defined.)

3. Radicals of semigroup rings over completely 0 -simple semigroups. If $D$ is a semigroup and $R$ is an associative ring, the semigroup ring of $D$ over $R$ is defined to be the set of all functions from $D$ into $R$ of finite support. We denote the semigroup ring of $D$ over $R$ by $R D$. Addition is pointwise and multiplication is convolution. Thus, if $x, y \in R D$, then

$$
(x+y)(d)=x(d)+y(d)
$$


and

$$
x y(d)=\sum_{a b=d} x(a) y(b) .
$$

Under these operations $R D$ is an associative ring. We write the elements of $R D$ as finite formal sums $x=\alpha_{1} d_{1}+\ldots+\alpha_{n} d_{n}$, where $x\left(d_{i}\right)=\alpha_{i} \in R$. If $D$ has a zero, the contracted semigroup ring, denoted by $\overline{R D}$, identifies the zero $z$ of $D$ with the zero of the semigroup ring. More specifically, we define $\overline{R D}$ to be the ring $R D / I$, where $I=\{x \in R D \mid x(d)=0$ if $d \neq z\}$.

The following lemma reduces the study of contracted semigroup rings of completely 0 -simple semigroups to the study of rings of the form $\mathscr{M}_{\rho \gamma}^{*}(T ; I, \Lambda ; P)$. We shall show (Lemma 3.9) that, for a wide class of properties $\pi$, if $R$ is $\pi$-semisimple, then $R D$ is $\pi$-semisimple if $\overline{R D}$ is.

Leмma 3.1. Let $D$ be a completely 0 -simple semigroup represented as a Rees matrix semigroup by $D=\mathscr{M}\left(G^{0} ; I, \Lambda ; P\right)$. Then the contracted semigroup ring $\overline{R D} \cong \mathscr{M}_{\rho \gamma}^{*}(R G ; I, \Lambda ; P)$.

Proof. Let $x \in \overline{R D}$; say, $x=\alpha_{1} d_{1}+\ldots+\alpha_{n} d_{n}, d_{i} \neq z$. Each $d_{i}$ is a Rees $I \times \Lambda$ matrix over $G^{0}$; suppose $d_{i}$ has $g_{i} \neq 0$ as its $\left(k_{i}, \lambda_{i}\right)$-entry and zero elsewhere. Map $\alpha_{i} d_{i}$ onto the element of $\mathscr{M}_{\rho y}^{*}(R G ; I, \Lambda ; P)$ with $\alpha_{i} g_{i}$ as its $\left(k_{i}, \lambda_{i}\right)$-entry and zero elsewhere. Extend this map linearly to all of $\overline{R D}$; thus $x$ maps into the sum of the images of $\alpha_{i} d_{i}(i=1, \ldots, n)$. This map is an isomorphism of $R D$ onto $\mathscr{M}_{p y}^{*}(R G ; I, \Lambda ; P)$.

Definition 3.2. Let $P$ be a $\Lambda \times I$ matrix over a ring $T$ and let $R=\mathscr{A}_{\rho y}^{*}(T ; I, \Lambda ; P)$. $P$ is cancellable with respect to $R$ if, for $A \in R, A \neq 0$ imples that $A P \neq 0$ and $P A \neq 0$.

REMARK. If $T$ is a finite-dimensional algebra over some field and $I$ and $\Lambda$ are finite sets, of cardinality $n$ and $m$ respectively, then $m \neq n$ implies that no $m \times n$ matrix is cancellable (see Clifford and Preston [4], p. 157). This is not the case if $I$ and $\Lambda$ are infinite.

Note also that the conditions $A P \neq 0$ and $P A \neq 0$ are not redundant. In the following lemma we use only the requirement that $A \neq 0$ implies that $A P \neq 0$. The significance of requiring $P A \neq 0$ will be seen later.

Lemma 3.3. Let $R=\mathscr{M}_{\rho \gamma}^{*}(T ; I, \Lambda ; P), S=M_{\rho}^{*}(T ; I, I)$. Then $R$ is a left $S$-module. Define $\phi: R \rightarrow S$ by $\phi(A)=A P$. Then

(i) $\phi$ is both a ring homomorphism and an $S$-module homomorphism of $R$ into $S$.

(ii) If $P$ is cancellable with respect to $R$, then $\phi$ is one-to-one and hence $R$ is isomorphic to a left ideal of $S$.

Proof. It was observed earlier that $C \in S, B \in R$ imply that $C B \in R$, and it is easy to check that $R$ is an $S$-module. Statements (i) and (ii) are immediate.

For cancellable $P$, Lemma 3.3 reduces the study of rings of the form $\mathscr{M}_{\rho \gamma}^{*}(T ; I, \Lambda ; P)$ to the study of left ideals in $M_{\rho}^{*}(T ; I, I)$, the ring of column bounded $I \times I$ matrices over $T$. The next lemma will permit us to apply the results on radicals of left ideals in arbitrary rings which we developed in Section 2. 
Lemma 3.4. Let $R=\mathscr{M}_{\rho \gamma}^{*}(T ; I, \Lambda ; P)$ and $S=\mathscr{M}_{\gamma}^{*}(T ; I, I)$.

(i) $P$ cancellable with respect to $R$ and $\mathfrak{X}\left(T_{T}\right)=\{0\}$ imply that $\mathfrak{Q}\left(R_{R}\right)=\{0\}$.

(ii) $\mathfrak{N}\left({ }_{T} T\right)=\{0\}$ implies that $\mathfrak{N}\left({ }_{S} R\right)=\{0\}$.

(iii) $\mathfrak{I}\left({ }_{R} R\right)=\{0\}$ implies that $\mathfrak{Y}\left({ }_{T} T\right)=\{0\}$.

Proof. (i) Let $B \in R, B \neq 0$. Since $P$ is cancellable, $P B \neq 0$. Let $b$ be a nonzero entry of $P B$ in the $(\lambda, \mu)$-position. Since $\mathfrak{N}\left(T_{T}\right)=\{0\}$, there exists $a \in T$ such that $a b \neq 0$. Let $A$ be the $I \times \Lambda$ matrix with $a$ in the $(1, \lambda)$-position and zero elsewhere. Then $A P B \neq 0$; hence $\mathfrak{V}\left(R_{R}\right)=\{0\}$. Statements (ii) and (iii) are proved similarly.

Recall that $\pi$ is a Z-property if every ring $T$ such that $T^{2}=\{0\}$ is a $\pi$-ring.

THEOREM 3.5. Let $\pi$ be a left inductive Z-property. Let $R=\mathscr{M}_{\rho y}^{*}(T ; I, \Lambda ; P)$ and $S=M_{\gamma}^{*}(T ; I, I)$. If $P$ is cancellable with respect to $R$ and $\mathscr{U}(T)=\{0\}$, then $\mathscr{U}(R)=\{0\}$ if and only if $\mathscr{U}(S)=\{0\}$.

Proof. $\mathfrak{V}\left({ }_{T} T\right)$ and $\mathfrak{N}\left(T_{T}\right)$ are ideals of $T$ which square to zero. Since $\mathscr{U}(T)=\{0\}$ and $\pi$ is a Z-property, we can conclude that $\mathfrak{U}\left({ }_{T} T\right)=\{0\}$ and $\mathfrak{Q}\left(T_{T}\right)=\{0\}$. Hence, by Lemma 3.4, $\mathfrak{W}\left(R_{R}\right)=\{0\}$ and $\mathfrak{T}\left({ }_{S} R\right)=\{0\}$. Now, by Lemma 3.3, $R$ is isomorphic to a left ideal of $S$ since $P$ is cancellable. Applying Theorem 2.6, we conclude that $\mathscr{U}(R)=\{0\}$ if and only if $\mathscr{U}(S)=\{0\}$.

This theorem and Lemma 3.1 effectively reduce the question of $\pi$-semisimplicity of a contracted semigroup ring of a completely 0 -simple semigroup to the question of $\pi$-semisimplicity of a ring $S$ of column bounded matrices over a ring $T$.

Lemma 3.6. Let $\pi$ be a left inductive Z-property. Let $S=M_{\gamma}^{*}(T ; I, I)$. Then any $\pi$-ideal of $S$ is contained in $M_{\gamma}^{*}(\mathscr{U}(T) ; I, I)$. Hence $\mathscr{U}(T)=\{0\}$ implies that $\mathscr{U}(S)=\{0\}$. Conversely if $\pi$ satisfies Condition 2.3, then $\mathscr{U}(S)=\{0\}$ implies that $\mathscr{U}(T)=\{0\}$.

Proof. Let $A$ be a $\pi$-ideal of $S$. Let

$$
\bar{A}=\{a \in T \mid a \text { is an entry in some matrix in } A\} .
$$

We shall show that

$$
T \bar{A} T=\left\{\sum_{i=1}^{n} t_{i} a_{i} t_{i}^{\prime} \mid t_{i}, t_{i}^{\prime} \in T, a_{i} \in \bar{A}, n=1,2,3, \ldots\right\} \subseteq \mathscr{U}(T) .
$$

If $T \bar{A} T=\{0\}$, the claim is clearly true. Hence we may assume that there exist $t, t^{\prime} \in T, a \in \bar{A}$, such that $t^{\prime} t^{\prime} \neq 0$. Let $L$ be the subset of $S$ consisting of matrices whose first column has entries from $T \bar{A} T$ and all other entries zero. $L$ is a nonzero left ideal of $S$. Now $A$ contains all matrices with entries from the ideal $T \bar{A} T$ of $T$. For, given any $a \in \bar{A}$, the matrix with $t_{i} a t_{i}^{\prime}$ as the $(k, j)$-entry and zero elsewhere is obtained by multiplying the matrix of $A$ in which $a$ appears, say as the $(l, m)$-entry, on the left by the matrix with $t_{i}$ in the $(k, l)$-entry and zero elsewhere and on the right by the matrix with $t_{i}^{\prime}$ as the $(m, j)$-entry and zero elsewhere. Adding finitely many such matrices produces any bounded matrix with entries from $T \bar{A} T$. In particular, $L \subseteq A$. Hence $L$ is a $\pi$-ring, since $\pi$ is left inductive. Now $T \bar{A} T$ is a homomorphic image of $L$ under the map which takes a matrix in $L$ into its first entry. Hence $T \bar{A} T$ is a $\pi$-ring and so a $\pi$-ideal of $T$. Thus $T \bar{A} T \subseteq \mathscr{U}(T)$. This implies that, if $J$ is the ideal generated 
by (and containing) $\bar{A}, J^{3} \subseteq \mathscr{U}(T)$. Hence the image of $J$ under the natural homomorphism $T \rightarrow T / \mathscr{U}(T)$ is a nilpotent ideal. But $\pi$ is a Z-property; so $T / \mathscr{U}(T)$, being $\pi$-semisimple, does not contain any nilpotent ideals. Thus $J \subseteq \mathscr{U}(T)$. Hence $\bar{A} \subseteq \mathscr{U}(T)$ and so $A \subseteq M_{\gamma}^{*}(\mathscr{U}(T) ; I, I)$.

Conversely, assume that $\pi$ satisfies Condition 2.3 and that $\mathscr{U}(S)=\{0\}$. Suppose $Q$ is a $\pi$-ideal of $T$. Let $\hat{Q}$ be that subset of $S$ consisting of matrices with first column entries from $Q$ and zero elsewhere. Then $\hat{Q}$ is a left ideal of $S$. Let $N$ be the ideal of $\hat{Q}$ consisting of all matrices with upper left-hand corner zero; then $Q \cong \hat{Q} / N$. Now $N^{2}=\{0\}$, so that $N$ is a $\pi$-ideal of $\hat{Q}$ and hence Condition 2.3 implies that $\hat{Q}$ is a $\pi$-ring. But $\pi$ is left inductive and so $S$ has no nonzero left $\pi$-ideals. Hence $\hat{Q}=\{0\}$ and thus $Q=\{0\}$. We have shown that $T$ has no nonzero $\pi$-ideals. Hence $\mathscr{U}(T)=\{0\}$. [1].

REMARK. The proof of the converse above was motivated by Amitsur's Theorem 6.3 in

THEOREM 3.7. Let $R=\mathscr{M}_{\rho y}^{*}(T ; I, \Lambda ; P)$. Let $\pi$ be a left inductive Z-property satisfying Condition 2.3. Then $\mathscr{U}(R)=\{0\}$ if and only if $\mathscr{U}(T)=\{0\}$ and $P$ is cancellable with respect to $R$.

Proof. Assume that $\mathscr{U}(T)=\{0\}$ and that $P$ is cancellable. By Lemma 3.6, $\mathscr{U}(S)=\{0\}$. Hence by Theorem $3.5, \mathscr{U}(R)=\{0\}$. Conversely, assume that $\mathscr{U}(R)=\{0\}$. If $P$ is not cancellable, then either $\mathfrak{A}\left({ }_{R} R\right)$ or $\mathfrak{Y I}\left(R_{R}\right)$ is not zero. Hence there is a nonzero ideal in $R$ which squares to zero. But this contradicts $\mathscr{U}(R)=\{0\}$, since $\pi$ is a Z-property; therefore $P$ is cancellable. Also $\mathscr{U}(R)=\{0\}$ implies that $\mathfrak{N}\left({ }_{R} R\right)=\{0\}$, since $\pi$ is a Z-property; hence, by Lemma 3.4(iii), $\mathfrak{N}\left({ }_{T} T\right)=\{0\}$. By part (ii) of the same lemma, $\mathfrak{I}\left({ }_{S} R\right)=\{0\}$. Since we have already shown that $P$ is cancellable, Lemma 3.3 implies that $R$ is isomorphic (as an $S$-module) to a left ideal of $S$. Hence, by Theorem 2.6(ii), $\mathscr{U}(S)=\{0\}$ and, by Lemma 3.6, $\mathscr{U}(T)=\{0\}$.

Remark. Condition 2.3 is only needed to prove that $\mathscr{U}(R)=\{0\}$ implies that $\mathscr{U}(T)=\{0\}$.

Theorem 3.7 is now applied to contracted semigroup rings.

THEOREM 3.8. Let $D$ be a completely 0-simple semigroup represented as a Rees matrix semigroup by $D=\mathscr{M}\left(G^{0} ; I, \Lambda ; P\right)$. Let $\pi$ be a left inductive Z-property satisfying Condition 2.3. Then $\mathscr{U}(\overline{R D})=\{0\}$ if and only if $\mathscr{U}(R G)=\{0\}$ and $P$ is cancellable with respect to

$$
\mathscr{M}_{\rho y}^{*}(R G ; I, \Lambda ; P) \text {. }
$$

Proof. By Lemma 3.1, $\overline{R D} \cong \mathscr{A}_{\rho \gamma}^{*}(R G ; I, \Lambda ; P)$. The result now follows immediately from Theorem 3.7 .

REMARK. The stated conditions are sufficient for $\mathscr{U}(\overline{R D})=\{0\}$ without assuming that $\pi$ satisfies Condition 2.3.

The following lemma shows the connection between the $\pi$-semisimplicity of $\overrightarrow{R D}$ and that of $R D$.

Lemma 3.9. Let $\pi$ be an H-property. Let $D$ be a semigroup with zero element $z$. Then

(i) $\mathscr{U}(\overline{R D})=\{0\}$ and $\mathscr{U}(R)=\{0\}$ imply that $\mathscr{U}(R D)=\{0\}$;

(ii) $\mathscr{U}(R D)=\{0\}$ implies that $\mathscr{U}(R)=\{0\}$. 
Proof. (i) $\overline{R D}=R D / I$, where $I$ is isomorphic to $R$. In general, for H-properties, if $A$ is an ideal of a ring $T$, then $\mathscr{U}(A)=\{0\}$ and $\mathscr{U}(T / A)=\{0\}$ imply that $\mathscr{U}(T)=\{0\}$. The result follows.

(ii) If $P$ is a $\pi$-ideal of $R$, then $\{x \in R D \mid x(z) \in P, x(d)=0$ if $d \neq z\}$ is an ideal of $R D$ isomorphic to $P$. obvious.

We shall continue to discuss the semisimplicity of $\overline{R D}$. The implications for $R D$ are

Theorem 3.8 permits the application of any of the known results about group rings to obtain corresponding results for semigroup rings. The theory applies to at least three of the four properties that were discussed in Section 2A. As was observed then, all of the properties satisfy the hypotheses of Theorem 3.8 except possibly nil.

Some examples of the application of Theorem 3.8 follow. There are others.

COROLlaRY 3.10. Let $D=\mathscr{M}\left(G^{0} ; I, \Lambda ; P\right)$. Then $\mathscr{P}(\overline{R D})=\{0\}$ if and only if

(i) $P$ is cancellable with respect to $\mathscr{M}_{\rho \gamma}^{*}(R G ; I, \Lambda ; P)$,

(ii) $\mathscr{P}(R)=\{0\}$,

(iii) the orders of the finite normal subgroups of $G$ are cancellable in $R$.

Proof. Simply apply Connell's result for group rings ([5], p. 162) and Theorem 3.8.

Corollary 3.11. Let $D=\mathscr{M}\left(G^{0} ; I, \Lambda ; P\right)$. Let $F$ be a transcendental extension of the rationals. Then $\mathscr{J}(F D)=\{0\}$ if and only if $P$ is cancellable with respect to $\mathscr{M}_{\rho \gamma}^{*}(F G ; I, \Lambda ; P)$.

Proof. Amitsur [3] has shown that under these conditions $\mathscr{g}(F G)=\{0\}$.

COROLlary 3.12. (Munn). If $F$ is a field and $D$ is a finite 0-simple semigroup,

$$
D=\mathscr{M}\left(G^{0}, m, n ; P\right),
$$

then $\overline{F D}$ is completely reducible if and only if $P$ is invertible regarded as a matrix over $F G$ (in particular $m=n$ ) and the characteristic of $F$ does not divide the order of $G$.

Proof. $\quad F G$ is a finite dimensional algebra over $F$. For $m \times n$ matrices over finite dimensional algebras, invertibility is equivalent to cancellability. (See [4], Corollary 5.10 and Theorem 5.11.) Also, since $\overline{F D}$ is finite dimensional over $F, \overline{F D}$ is completely reducible if and only if $\mathscr{F}(\overline{F D})=\{0\}$. Applying Theorem 3.8, we see that $\overline{F D}$ is completely reducible if and only if $\mathscr{f}(F G)=\{0\}$ and $P$ is invertible. But, by Maschke's Theorem, $\mathscr{J}(F G)=\{0\}$ if and only if the characteristic of $F$ does not divide the order of $G$. The result follows.

We have not investigated the question of which completely 0 -simple semigroups have cancellable sandwich matrices $P$. This question deserves some study. For a particular class of semigroups, however, the answer is easy. Brandt semigroups are defined in [4]. A semigroup $D$ is inverse if, for every $a \in D$, there exists a unique $x$ such that $a x a=a$ and $x a x=x$. Either of the following conditions is necessary and sufficient for a semigroup $D$ with zero to 
be a Brandt semigroup:

(i) $D$ is a completely 0 -simple inverse semigroup,

(ii) $D$ is isomorphic to a Rees $I \times I$ matrix semigroup $\mathscr{M}\left(G^{0} ; I, I, \Delta\right)$ over a group with zero $G^{0}$ with the sandwich matrix $\Delta$ equal to the $I \times I$ identity matrix. (Cf. [4], Theorem 3.9.)

THEOREM 3.13. Let $D$ be a Brandt (completely 0-simple inverse) semigroup with a maximal subgroup G. Let $\pi$ be a left inductive Z-property satisfying Condition 2.3. Then $\mathscr{U}(\overline{R D})=\{0\}$ if and only if $\mathscr{U}(R G)=\{0\}$.

Proof. The Rees structure theory shows that $D \cong \mathscr{M}\left(G^{0} ; I, I, \Delta\right)$ where $G$ is a maximal subgroup. The result follows from Theorem 3.8, since the identity matrix is always cancellable.

REMARK 3.14. Although Theorem 3.8 cannot be applied to the nil radical, it is possible to obtain special results concerning the existence of one sided nil ideals in $\overline{R D}$. It can be shown that, if $D=\mathscr{H}\left(G^{0} ; I, \Lambda ; P\right), P$ is cancellable with respect to $\mathscr{M}_{\rho \gamma}^{*}(R G ; I, \Lambda ; P)$ and $R G$ has no nil left ideals, then $\overline{R D}$ has no nil left ideals.

\section{REFERENCES}

1. S. A. Amitsur, A general theory of radicals, II, Amer. J. Math. 76 (1954), 100-125.

2. S. A. Amitsur, A general theory of radicals, III, Amer. J. Math. 76 (1954), 126-136.

3. S. A. Amitsur, On the semi-simplicity of group algebras, Michigan Math. J. 6 (1959), 251-253.

4. A. H. Clifford, and G. B. Preston, The algebraic theory of semigroups, Volume 1, Math. Surveys of the American Math. Soc. 7 (Providence, R.I., 1961).

5. J. Lambek, Lectures on rings and modules, (Waltham, Massachusetts 1966).

6. W. D. Munn, On semigroup algebras, Proc. Cambridge Philos. Soc. 51 (1955), 1-15.

7. E. M. Patterson, On the radicals of certain rings of infinite matrices, Proc. Roy. Soc. Edinburgh Sect. A. 65 (1961), 263-271.

8. D. Rees, On semi-groups, Proc. Cambridge Philos. Soc. 36 (1940), 387-400.

UNIVERSITY OF WISCONSIN

AND

University of California, Santa Barbara 